\title{
Introducing astronomy: a way to renovate school curricula
}

\author{
Vira Godunova \\ ICAMER Observatory, NAS of Ukraine, \\ 27 Zabolotnoho Str., 03680 Kyiv, Ukraine \\ email: godunova@mao.kiev.ua
}

\begin{abstract}
One of the most urgent tasks facing society today is to make science more attractive to the younger generation and to foster the continuous improvement of the knowledge and skills. These objectives assume ever greater importance because, firstly, young people are opting less and less for scientific studies, secondly, we need to establish new starting points for them in order that they could better understand and improve our changing world. To achieve these aims, it is necessary to expand innovation in secondary education. In this paper, a few suggestions, which can help assess and prioritize the usefulness of astronomy-related material for improving the educational process, are presented.
\end{abstract}

Keywords. School curricula, interdisciplinary connections, science education, teaching, history, literature, geography

\section{Introduction}

Today, many young people have a limited view of the possible advantages of being a scientist. Only a small part of them prefer book learning. How can we make science most interesting to them? Since school curriculum is traditionally based on well-established science that often is somewhat tedious it is important to make lessons interesting early on, for instance, by using examples and applications from astronomy. Most every discipline can benefit from inclusion of astronomy-related material because the fascinating world of astronomy is an effective means to arouse greater interest of young people in science and to develop their abstract thinking that is necessary to connect everyday life to the complexity of the world.

This approach is not intended to compete with standard methods; rather, it offers a more interactive style of teaching.

\section{A twofold strategy for success}

Evidence-based and inspiring information on astronomical discoveries and space exploration can stretch young minds and foster their understanding of the world. How might this be achieved in practice? The approach includes the following:

- providing intriguing information, e.g. alternative explanations of phenomena;

- referring to striking examples;

- presenting images obtained by ground- and space-based instruments.

On the other hand, astronomy may help teachers to present a full picture of their disciplines, whether of the natural sciences or the humanities. There are a lot of interesting facts and phenomena related to astronomy, which could be mentioned in lessons in physics, biology, geography, history, and even literature. Some of the most exciting examples in this field are given below. 


\subsection{Preserving the promise and inspiration in school subjects}

History. For instance, giving lessons in history, teachers might mention the following interesting facts, which are just a few of many related to astronomy:

- the Maya, as well as the ancient Egyptians used precise calendars; their astronomical skills included the Sun's and planets' cycles and location of stars in the sky;

- there exists an opinion that the Giza pyramid complex is a sky-map of the Belt of Orion. By the ancient Egyptians, the stars of this constellation were associated with Osiris, the god of death and underworld.

Literature. It is well known that many of famous writers and poets described in their works celestial objects and phenomena. In this connection, Gulliver's Travels, a prose satire by Jonathan Swift, deserves special attention. In this 1726 book, Swift described a fictional place, the flying island Laputa, whose population mainly consisted of educated people. They have made great strides in research and technology, especially in astronomy:

"They spend the greatest part of their lives in observing the celestial bodies, which they do by the assistance of glasses, far excelling ours in goodness. For, although their largest telescopes do not exceed three feet, they magnify much more than those of a hundred with us, and show the stars with greater clearness. This advantage has enabled them to extend their discoveries much further than our astronomers in Europe; for they have made a catalogue of ten thousand fixed stars, whereas the largest of ours do not contain above one third part of that number. They have likewise discovered two lesser stars, or satellites, which revolve about Mars; whereof the innermost is distant from the centre of the primary planet exactly three of his diameters, and the outermost, five; the former revolves in the space of ten hours, and the latter in twenty-one and a half; so that the squares of their periodical times are very near in the same proportion with the cubes of their distance from the centre of Mars; which evidently shows them to be governed by the same law of gravitation that influences the other heavenly bodies.

They have observed ninety-three different comets, and settled their periods with great exactness. If this be true (and they affirm it with great confidence) it is much to be wished, that their observations were made public, whereby the theory of comets, which at present is very lame and defective, might be brought to the same perfection with other arts of astronomy."

There is a startling fact: in reality, the two satellites of Mars were discovered in 1877, i.e. 150 years after publication of Swift's bestseller! Moreover, their characteristics (apogee altitudes, rotational periods, etc.) reported in Gulliver's Travels compare favorable to modern data. It is a real mystery how did Swift know about Martian moons long before they were discovered.

Geography. In geography, the synthesis of data from many disciplines is the key to better understanding complex Earth-system phenomena, as well as geological and atmospheric processes. Such an approach provides an interesting framework for studying the Earth by comparing with other planets. Large ground-based telescopes and space missions revealed many striking things about the Solar System objects. Teachers can fit into their classes the following material (images and information) relating to specific topics:

- Most planets and some of their satellites have atmospheres (and even meteorology, as in the case of the Saturn's giant moon Titan (Lorenz \& Mitton 2008). But the composition of those atmospheres is sufficiently different from that Earth's, beneath them lie barren worlds. However, the surfaces of those celestial bodies in some areas look like on Earth. There are, for instance, similar landscapes, as photographed on Earth (Hawaii), Mars, and Titan, respectively (Fig. 1).

- Craters, volcanoes, and mountains cover the surfaces of many solid Solar System 
objects. For example, the mount Olympus Mons on Mars is $27 \mathrm{~km}$ high. For comparison, the highest mountain on Earth, Everest, stands only 8,848 meters above see level.

- Volcanism is widespread in the solar system. Active volcanoes are present on the moons of the outer planets of the solar system (Sotin 2008). Images of volcanic eruptions on Jupiter's moon Io recorded by cameras aboard the Voyager 1 spacecraft are most impressive.

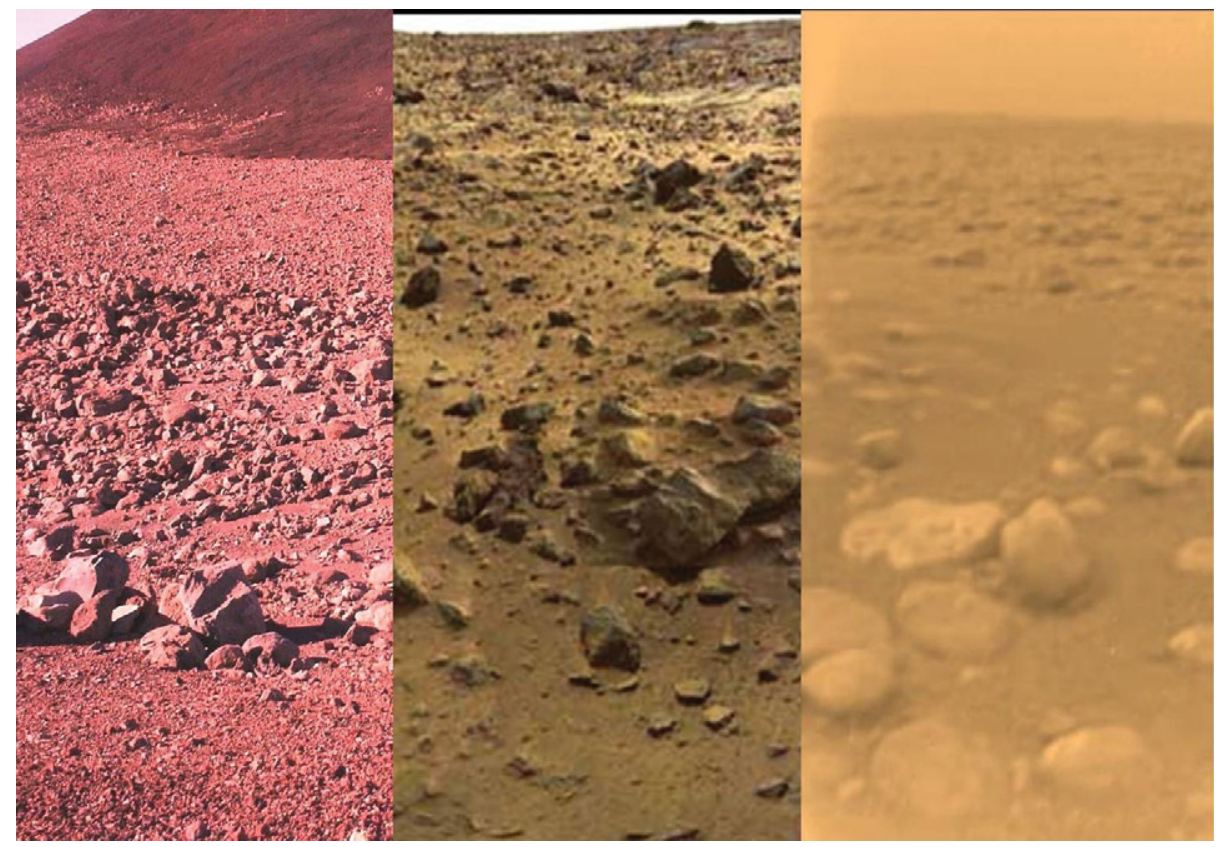

Figure 1. Comparison of volcanic landscapes in Hawaii, Mars and Titan (from left to right).

\subsection{Developing analytical skills and imagination}

Teachers can help pupils to develop their own opinions by providing them with additional, often intriguing information, rather than only focusing their attention on inspiring astronomical phenomena. An early introduction to the basics of scientific thinking plays an important rôle in engaging and preparing young people to pursue science and engineering.

Biology. It is necessary to bear in mind that the strong science does not always provide the answer to a problem. For instance, biology is closely tied to the theory of origin and evolution of life on Earth. In the school curriculum, the Oparin's hypothesis is adopted, according to which life emerged from the inanimate matter via a spontaneous process of prebiotic molecular evolution. However, it is appropriate to mention here that there exist quite different views on the origin of life on Earth. One of them, the panspermia hypothesis, is that life was brought to Earth from outer space. The matter is that microorganisms could be transported over long distances in space inside natural vehicles such as molecular clouds, comets or meteors. Recent studies on some meteorites have revealed biologically relevant structures, which might be of a non-terrestrial origin (Becker et al. 1999). Therefore, it seems rather probable that comets and meteorites could supply Earth with the seeds of life. Moreover, if the general conditions, which made possible life on Earth, are not exceptional, similar conditions can arise on planets around other stars. This suggests that life may be ubiquitous in the Universe. Thus, 
using the abovementioned material in their lessons in biology, teachers engage audiences in question posing and emphasize gathering evidence and testing alternative explanations.

Physics. Lessons might present some topics in more depth in an effort to build breadth of understanding across different disciplines. Such a rational learning can be illustrated by the following example. Gravitation is a fundamental force in the universe. Every planetary body (including the Earth) has its own gravitational field, which exerts an attractive force on all objects. The strength of this field at any given point is proportional to the planetary body's mass. Gravity rules our life on Earth, we almost never notice it. But what is about gravity on other celestial bodies? In the not too distant future, manned missions to other planets could become reality. People are thinking now in terms of discovering new Earths orbiting foreign, distant stars. But new-found exoplanets are characterized by a large mass and diameter, and, therefore, by larger gravitation, which is critical for humans. If that is so, how can mankind explore others worlds? A discussion by considering the problem of gravity leads to other issues of space exploration, especially to innovative activity in the field of robot technology.

\section{Conclusions}

The inclusion of astronomy-related material in various school disciplines can help rebuild the children's impression of science that looks too hard. Such approach is especially aimed at developing innovative thinking and attracting young people to the scientific and technical career fields.

The following steps could be taken in order to overhaul the school curricula:

- developing new didactic materials in order to use astronomy-related facts and phenomena in teaching different school disciplines;

- nvolving professional astronomers in preparing relevant materials;

- promoting scientists to give lectures at schools that could become an important factor in enhancement of educational attainments in the sciences.

\section{Acknowledgements}

I would like to thank Dr. Theodor Kostiuk, NASA GSFC, for his early comments and help.

\section{References}

Becker, L., Popp, B., Rust, T., \& Bada, J.L. 1999, Earth Planet. Sci. Lett., 167, 71

Lorenz, R.D., \& Mitton, J. 2008, Titan Unveiled: Saturn's Mysterious Moon Explored (Princeton: Princeton University Press)

Sotin, C. 2008, American Geophysical Union, Fall Meeting 2008, abstract \#MR54A-01 